\title{
CASE REPORT OF RUPTURED NON-COMMUNICATING RIGHT RUDIMENTARY HORN PREGNANCY: AN ACUTE EMERGENCY
}

\author{
Che Hasnura Che Hassan, Abdul Kadir Abdul Karim, Nor Azlin Mohamed Ismail, Mohd Hashim Omar
}

Department of Obstetrics \& Gynaecology, Faculty of Medicine, Universiti Kebangsaan Malaysia, Jalan Yaacob Latiff, 56000 Cheras, Kuala Lumpur, Malaysia

Summary: Ruptured pregnancy in the rudimentary horn of women who have had a vaginal delivery is rare and unpredictable. However, when undiagnosed, this condition could lead to maternal morbidity and mortality. We report a pregnancy at 19 weeks gestation presented with acute abdomen and hypovolemic shock. She was initially thought to have an intrauterine pregnancy with the provisional diagnosis of a ruptured uterus. Intraoperatively, a ruptured non-communicating right rudimentary horn with ex utero pregnancy was discovered.

Key words: Rudimentary horn pregnancy; Ruptured; Abdominal pregnancy

\section{Introduction}

Unicornuate uterus is a congenital uterine anomaly resulting from a non-developing Mullerian duct or agenesis of the Mullerian system. It was first classified in 1979 by Buttram (2) and Gibbons and further revised by the American Society of Reproductive Medicine in 1988 (6). It is a Type II classification with unilateral hypoplasia or agenesis that can be further subclassified into communicating, noncommunicating, no cavity and no horn. Unicornuate uterus is thought to occur in the general population at a rate of 1 in 4020 and about $84 \%$ of these cases have a contralateral rudimentary horn $(4,5)$. Ruptured pregnancy in a rudimentary horn is an emergency which may result in morbidity and mortality. In women who have previously had vaginal deliveries, this problem is not at all expected.

\section{Case Reports}

A 28-year-old G2 P1 woman at 19 weeks period of amenorrhea (POA) presented with severe abdominal pain 4 hours prior to admission. Upon arrival, she was noted to be in hypovolemic shock with severe pallor, hypotension and tachycardia. The abdomen was tense, with the Symphysiofundal height of 24 weeks' size. No vaginal bleeding was noted. The initial impression was a concealed abruptio placenta. One month prior to this visit, she had presented for threatened miscarriage at 15 weeks POA but was told the pregnancy was normal. She had a past obstetric history of preterm delivery at 35 weeks POA. She delivered a $2.3 \mathrm{~kg}$ baby girl vaginally without any other complications. No other significant history was noted.

Further assessment with transabdominal ultrasound showed a nonviable fetus at 17 to 18 weeks' size with anhy- dramnios. The placenta was posterior low lying posteriorly with no retroplacental clots seen. In view of the previous sonographic evidence of an intrauterine pregnancy during her earlier visit, a provisional diagnosis of a ruptured uterus with a differential diagnosis of an abdominal pregnancy was made. An emergency laparotomy was performed immediately after 30 minutes of resuscitation. Intraoperative

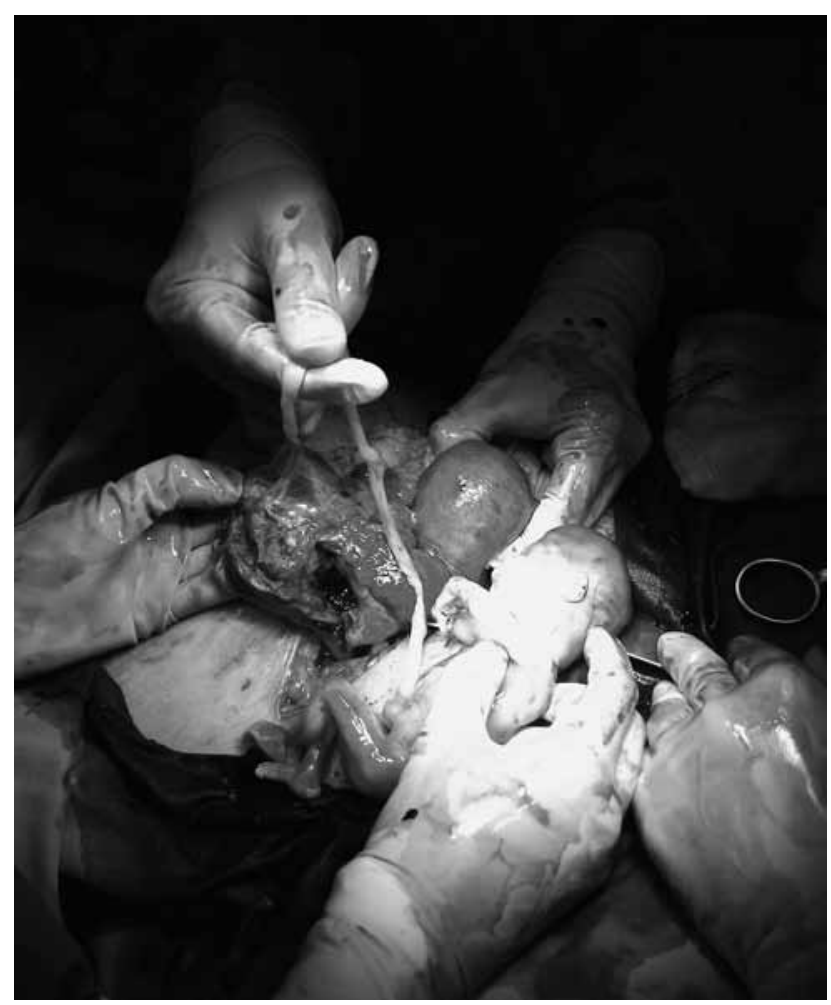


findings revealed a unicornuate uterus with ruptured right rudimentary horn pregnancy with the fetus over the right side of the abdomen. The placenta was attached to the cavity of the rudimentary horn (figure 1).

It was, however, difficult to visualize the uterine septum. No communication from the horn to the contralateral uterine cavity was seen. Both the ovaries and the fallopian tubes were normal. The right horn and tube were removed together with the nonviable fetus within. A total volume of 3.5 liters of haemoperitoneum blood was removed intraoperatively. Due to the significant blood loss throughout the operation, the patient was given a massive packed cell blood transfusion of 5 pints and one cycle of disseminated intravascular coagulopathy (DIVC) regime. Both the ureters and kidneys were normal. Post-operatively, the patient was transferred to a high dependency unit. Her post-operative hemoglobin level was $9.8 \mathrm{~g} / \mathrm{dL}$.

She was discharged healthy on the 5 th post op day after proper contraceptive advice. She was scheduled for a hysterosalphingogram 6 weeks post-operatively before any further decisions on future pregnancy.

\section{Discussion}

The incidence of uterine anomalies ranges between $0.5 \%$ and $2 \%$. Unicornuate uterus (Type II) can further be classified as those having an endometrial cavity and those with no endometrial cavity, and $90 \%$ of the cases are those with an endometrial cavity. A further division can be between the communicating and non-communicating types. Identification of unicornuate uterus is almost always accidental, as it is usually asymptomatic until reproductive age. Initial presentation often takes place with a recurrent first trimester miscarriage (5-10\%), a second trimester loss (25\%) (1), or during an infertility work-out.

In cases with successful pregnancy, the risks of recurrent early trimester loss and mid-trimester loss are increased, with preterm labour and abnormal fetal lie contributing factors. Taking our patient's previous pregnancy history, a premature delivery at 35 weeks could have been an indicator of uterine anomaly. Pregnancy in a rudimentary horn is a form of ectopic pregnancy and it can cause mortality or severe morbidity. It can occur in both communicating and non-communicating types. In the case of a non-communicating rudimentary horn like our patient had, it is postulated that the fertilization was possibly due to transperitoneal migration of the sperm (4).
The outcomes of pregnancy in rudimentary horns are either ectopic pregnancy $(2.7 \%)$, first trimester loss $24.3 \%$, second trimester abortion $9.7 \%$, preterm delivery $20.1 \%$, intrauterine demise (10.5\%) and $49.9 \%$ live birth up to $28-30$ weeks of gestation (5). Uterine rupture in the second trimester is usually a life-threatening condition resulting from a much thinner myometrial part of the rudimentary horn, with the non-functional endometrium leading to placenta adherence anomalies.

In our patient, even though she presented early with threatened miscarriage, the diagnosis of ectopic pregnancy was only established when she went into hypovolemic shock at 19 weeks. Sensitivity in detecting rudimentary horn uterus through ultrasound is only $30 \%$ and the condition is commonly missed (3). The non-communicating rudimentary horn rupture was only confirmed intraoperatively and removal of the rudimentary horn and ipsilateral fallopian tube was done. This was to reduce the risk of the patient having another ectopic pregnancy in the future and could also reduce the risk of dysmenorrhea (as a result of accumulated blood during menstrual cycle and retrograde menstruation). As $31 \%$ of patients with mullerian anomalies will also have urinary anomalies with congenital absence of a kidney (2), it is mandatory for this woman to have further assessment before attempting any future pregnancy.

In conclusion, although an ectopic pregnancy in a non-communicating rudimentary horn is uncommon, the diagnosis is a great challenge. The risk for future ectopic pregnancy and uterine rupture are still present, thus the need for vigilant care and obstetrics expertise to prevent devastating problems.

\section{References}

1. Acien P. Incidence of mullerian defects in fertile and infertile women. Hum Reprod 1997; 12:1372-6.

2. Buttram V, Gibbons W. Mullerian anomalies: a proposed classification from analysis of 144 cases. Fertil Steril 1979; 32:40-6.

3. Chopra S, Keepanasseril A, Rohilia M, et al. Obstetric morbidity and the diagnostic dilemma in pregnancy in rudimentary horn: retrospective analysis. Arch Gynecol Obstet 2009; 280:907-910.

4. Nahum G, Stanislaw H, McMohan C. Preventing ectopic pregnancies: How often does transperitoneal transmigration of sperm occur in effecting human pregnancy Br J Obstet Gynecol 2004; 111:706-14.

5. Reichman D, Laufer MR, Robinson BK. Pregnancy outcomes in unicornuate uteri: a review. Fertil Steril 2009; 91:1886-94.

6. The American Fertility Society. The American Fertility Society Classification of adnexal adhesions, distal tubal occlusion, tubal occlusion secondary to tubal ligation, tubal pregnancies, Mullerian anomalies and intrauterine adhesions. Fertil Steril 1988; 49:944-55.

Received: 09/03/2011

Accepted in revised form: 16/05/2011

\section{Corresponding author:}

Dr M I Nor Azlin, Department of Obstetrics \& Gynaecology, Faculty of Medicine, Universiti Kebangsaan Malaysia, Jalan Yaacob Latiff, 56000 Cheras, Kuala Lumpur, Malaysia; e-mail: azlinm@ppukm.ukm.my 